\title{
The Multi-agent System a Key Pillar in e-assessment for a real Competency Mapping
}

\author{
AichiYassir $^{1 *}$, Bassiri Mustapha ${ }^{2}$, Said Belaaouad $^{1}$, Said Benmokhtar $^{1}$ \\ ${ }^{1}$ Laboratory of Physical Chemistry of Materials, Ben M'Sik Faculty of Sciences \\ Hassan II University of Casablanca, BP 5366 Maarif, Casablanca, Morocco \\ yassiraichi@gmail.com
}

${ }^{2}$ ENS Casablanca, Hassan II University of Casablanca

Morocco

ABSTRACT: The e-assessment is a subject that has not ceased to arouse the interest of researchers in any field. Our study focuses on the architecture and modeling of e-assessment platforms and the impact of the multi-agent system on talent measurement. We define a metamodel describing the operating paradigm of this tool. The peculiarity of our application framework lies in the study of the last four ITEF master classes at Hassan II University. For this study, we used the "Innermetrix" tool, an e-assessment platform ranked number 1 worldwide.

The objective of this article is to have real competency mapping by defining a digital student repository associated with a digital study agent able to test and evaluate the soft skills of students in terms of output. The objective is to evaluate the cognitive-behavioral dimensions of students after spending two years in a training that combines a theoretical component and another practice.

Keywords: E-Assessment, Multi-Agent System (MAS), Competency Mapping, Innermetrix, Soft Skills

Received: 1 June 2019, Revised 10 August 2019, Accepted 22 August 2019

DOI: $10.6025 / \mathrm{jic} / 2019 / 10 / 4 / 128-136$

(C) 2019 DLINE. All Rights Reserved

\section{Introduction}

To secure decision-making and optimize the skills assessment process, various organizations use e-assessment. This tool is considered as a digital device that allows to map and measure $360^{\circ}$ degrees of skills using a range of tools, adapted to each case, including aptitude tests, an inventory of personality, one or more put in professional situations.

This skill mapping can't take place without resorting to a multi agent system (MAS). This system is based on autonomous virtual entities, based on a sequence of correlations between personality traits predefined by the evaluator, each correlation 
sequence called agent or artificial robot. Our goal was the integration of a digitized student repository, associated with a number of agents able to interact with each other, to measure and map the skills of students.

In order to guarantee better measurement reliability, we opted for the Innermetrix platform, the preferred e-assessment platform of major American organizations. This platform is based on the Hartman theory, a trend that has turned the science of axiology into a true measure of intrinsic values.

In this perspective, we have chosen the he last four master's promotions from ITEF (Training Engineering \& Technology of Education) master classes as a target audience in order to map and measure the soft skills of candidates after two years of training, and to take advantage of the data obtained to propose areas for improvement to the teaching staff.

\section{The Review of Literature}

In order to prepare our study, we relied on several sources to construct this part, the main one being (Wooldridge, 2001). Michael Wooldridge is, to our knowledge, one of the only ones to have completed a thorough history of the field as an appendix to his book. Our goal is to sketch a synthetic overview of the people, works and main advances behind the development of multi-agent systems as we know them today.

The multi-agent Systems are a multidisciplinary field that has its origins in many areas not limited to pure computer science, the humanities have brought a lot. We find references in artificial intelligence, competing systems, through game theory, logic, language or sociology, cognitive science. The multi-agent Systems are a multidisciplinary field that has its origins in many areas not limited to pure computer science, the humanities have brought a lot. We find references in artificial intelligence, competing systems, through game theory, logic, language or sociology, cognitive science.

The multidisciplinary of the field makes its study complex, so it would be illusory and pretentious to want to draw an exhaustive landscape of multi-agent systems. We will try instead to focus on the big dimensions of numerical evaluation, according to an empirical-inductive approach. The analysis of the literature also allowed us to identify the following elements: The taxonomy of agents; Modeling interactions and exchanges between agents \& Architecture and global modeling of e-assessment platforms.

\subsection{The Taxonomy of Agents}

The set of architectures agree on the fact that an agent receives (or perceives) flows (or stimuli) from its environment via sensors (or sensory organs), proceeds to a processing on these data and restores an action on the through its effectors (or organs engines) (1). These actions are themselves perceived by the agent, this forming a loop: the agent is described as being integrated into its environment (embedded according to (Ferguson, 1992)). The architectures are numerous but each one aims to provide the agent with a certain level of autonomy and intelligence in the information processing phase, and consequently in the acuity of these decisions materialized by his actions on the environment (2).

\begin{tabular}{|l|l|l|}
\hline & Cognitive agents & Reactive agents \\
\hline Teleonomic behavior & Intentional agents & Driven agents \\
\hline Reflex behavior & "Modules" Agents & Tropical agents \\
\hline
\end{tabular}

Table 1. Operating Paradigm of an Agent

\subsection{Modeling Interactions and Exchanges between Agents}

The description of what is an agent, or rather a multi-agent system, can't be complete without the fundamental aspect of the interactions that an agent can maintain with other entities of his environment, be it agents or mere objects. The agent itself is not enough. These are the interactions he will have with his environment and especially with other agents who has an interest. In this part, we will present the principle of communication between agents by sending messages, standardized languages today to implement this type of communication and communication procedures allowing the existence of social behavior. This communication process is essential, especially in the context of behavioral simulation. Agents interact through a set of events 


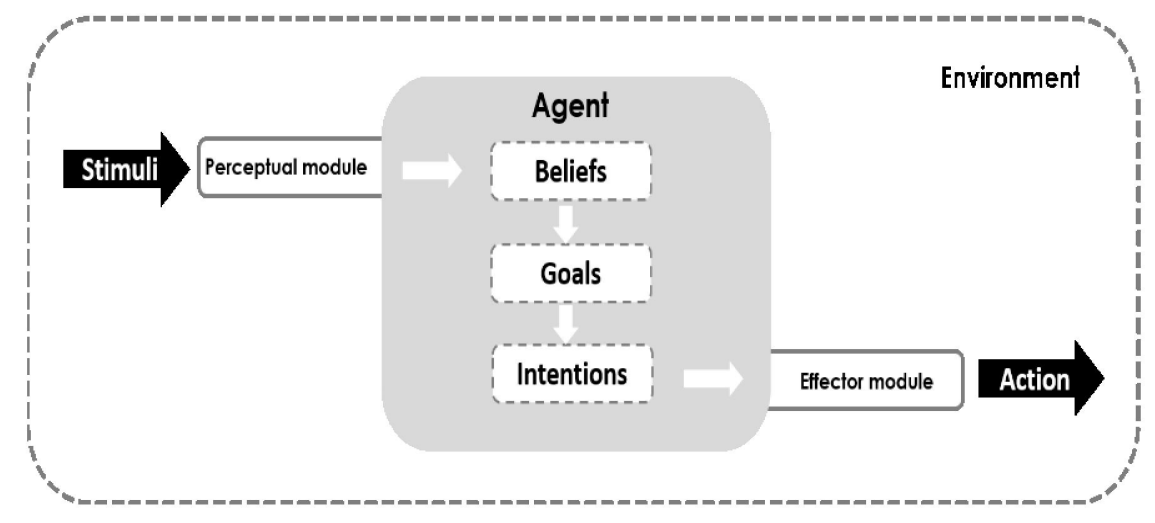

Figure 1. Operating Paradigm of an Agent

during which agents interact with each other, either directly or through the environment (3).

The set of interactions and exchanges forms an autonomous entity called a hybrid agent (4). In an object context, the interactions consist in exchanging information for the simple purpose of enriching each other's knowledge, at the higher level we can find communication processes allowing one entity to solicit another one, then processes allowing the synchronization tasks performed by several entities in parallel and finally processes allowing a set of entities to collaborate to perform one or more tasks.

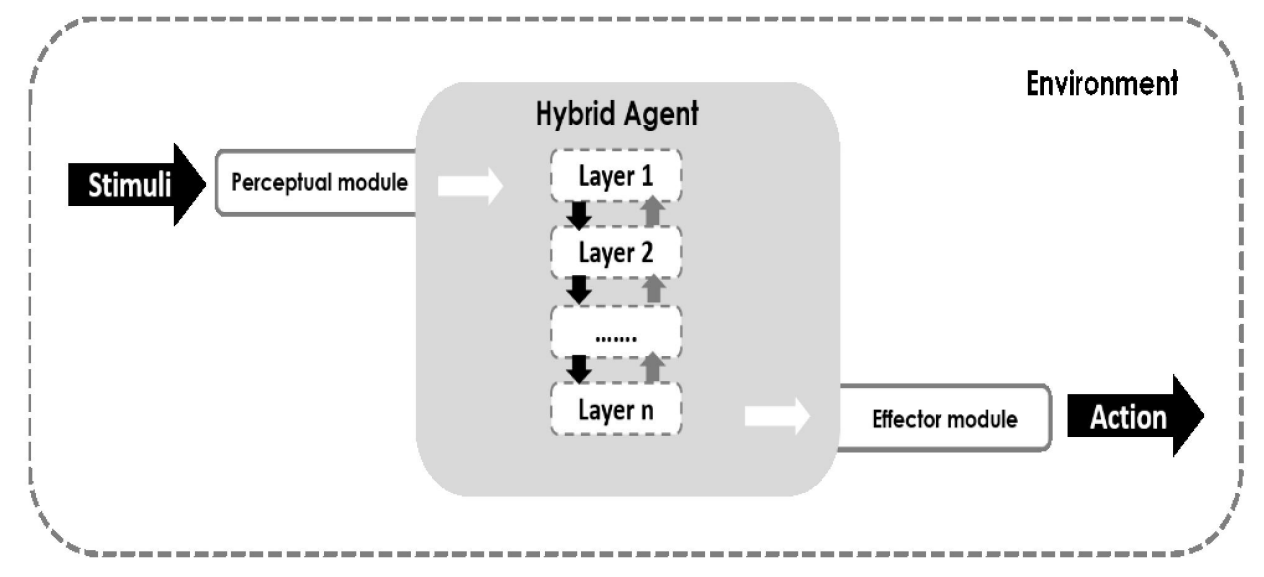

Figure 2. Modeling interactions and exchanges between agents

\subsection{Architecture and Global Modeling of e-assessment Platforms}

In "An Ontology-based Approach to Student Skills in Multi-Agent E-Learning Systems" (Gladun, 2007), Gladun and Rogushina propose to build an optimal metamodel, by field of competence, to improve the efficiency of a system E-Learning made available to students (5). As part of our study we propose a similar metamodel for the definition of the global architecture of an eassessment system.

The metamodel offers a solution to solve planning problems based on multi-agent technologies. Agents are able to deal with problems in particular areas and federate with each other to solve a larger problem (6). To do this, they join negotiating groups in which they express their intentions, exchange their respective goals and conditions of realization (7). There is then a comparison of the various proposals of the agents with the release of a consensus and constitution of a schedule of concatenation of these propositions.

The article introduces a development framework, a dedicated inter-agent communication protocol and a method for developing a target schedule from the negotiated proposals. The negotiation is inspired by Jin \& Koyama's work in "Multiagent planning through expectation-based negotiation" (Jin, 1990) (8). An agent joins the bargaining group only if the exchanges in question 
affect their interests and if the pre-conditions for achieving their goal are met.

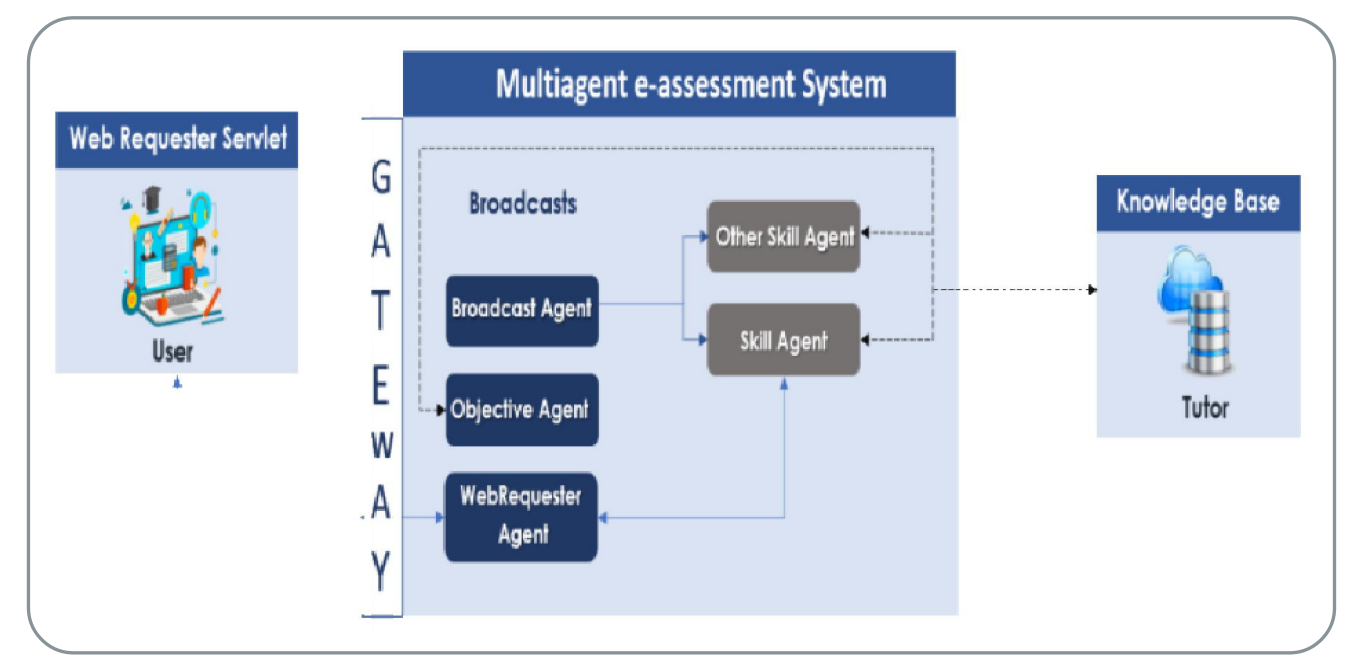

Figure 3. Architecture and global modeling of e-assessment platforms

\section{Materials and Methods}

In a multi-agent system, an agent with learning functions analyzes the variations of his environment, adapts and reacts according to the objective set by the evaluator. This triggers a behavior adapted to the typology of the variations observed. The objective of this learning consists, for an agent (alone or in group), to reach fixed or non-fixed objectives (thus potentially evolutionary). The achievement of these objectives is represented by the determination of a "policy" or a "strategy".

The strategy adopted in this article is to have a thorough knowledge of the student before starting his professional life, which will allow us to define and map with great precision the strengths and areas for improvement. To achieve this goal, we have chosen the Innermetrix electronic evaluation platform as a tool for measuring skills. The particularity of this platform is that it allows us to synchronize and develop a student repository, as well as the performance of its multi-agent system allowing us to obtain a correct mapping of skills. The choice of competencies to be evaluated puts more emphasis on the interest of the university as a training and learning institution and what it offers as a profile to the professional market.

The Innemetrix tool categorizes the behaviors of the individual according to two axes, with a passive or active action orientation, depending on whether the person perceives his environment as favorable or unfavorable (9). By placing the axes at right angles, four quadrants are formed, each describing a pattern of behavior. He called this disc theory. The four dimensions of behavior in DISC models are:

- Dominance: Active positive actions undertaken in an antagonistic / unfavorable context environment.

- Influence: Active positive actions taken in a supportive environment.

- Submission: Passive actions taken in a favorable environment.

- Compliance: Passive actions (aimed at reducing antagonistic factors) undertaken in an antagonistic / unfavorable environment.

\section{These four dimensions are described in the following figure:}

The four quadrants create clusters of behavioral tendencies resulting from the combination of the four dimensions of behavior (10). Each person will demonstrate some of the behavior for each dimension, but each person will develop their own unique combination of intensity or frequency for each of the four dimensions.

In the DISC model:

- The two upper quadrants (D and I) are extroverted and active, seeking to modify, control, influence or shape their environment 


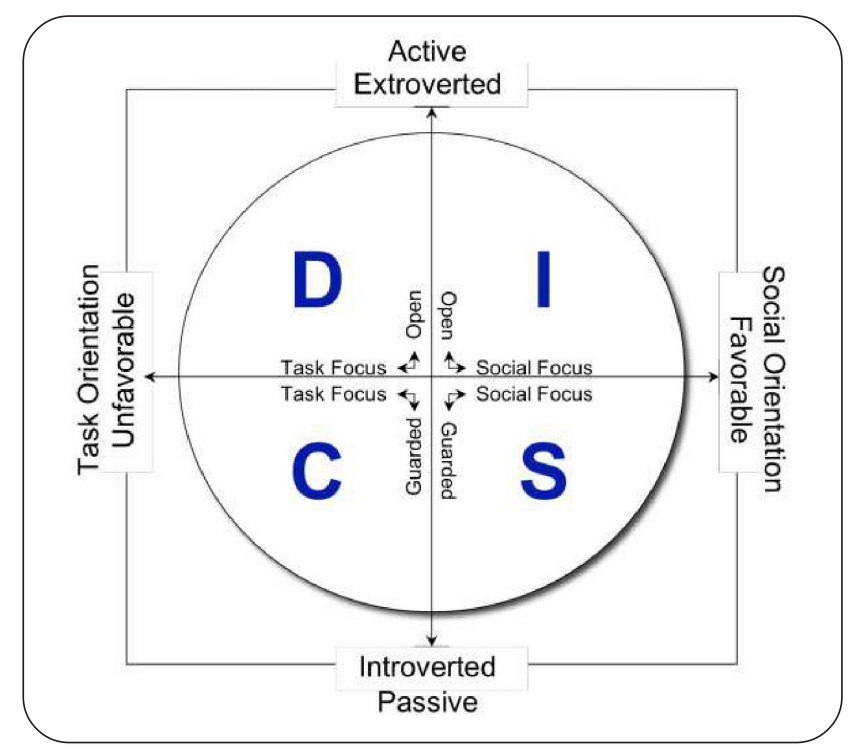

according to their particular vision. They are individuals who focus on what's more than just how or why and who continually challenge and test the boundaries of the environment and look for new ways.

- The two lower quadrants ( $\mathrm{S}$ and $\mathrm{C}$ ) are passive and introverted, seeking to focus on the why and how of a situation and, instead of trying to change the existing environment, are more interested in protect it or continue it.

The DISC Index is based on the same design as the previous DISC instruments, but has been updated to further utilize the power of the MAS (11). The DISC Index instrument allows the online respondent to click and drag the four instructions a ranking that represents the feeling of "more like me" to "less like me". The addition of this ranking instrument is a first in the university, to the knowledge of the research team, and greatly improves the design of this instrument by allowing the respondent to create a real hierarchy reflecting his true feelings (12). Most importantly, it allows the respondent to give $100 \%$ more information. By assigning a value to the four elements instead of only half, the respondent has greater precision in its ability to represent its true image of its behavioral preferences. Each respondent receives fourteen sets of selections or four-way rankings.

The respondent's ranks corresponding to the fifty-six (56) items are used to construct the four DISC scales: determination, interactivity, stability and caution. The relative value of each dimension is then plotted in two separate graphs, one representing the natural style of the respondent, the other its adaptive style. Each graph is accompanied by an individual descriptive text describing certain aspects of the respondent, including:

- Main Features

- Strengths

- Weaknesses

- The motivations

- Culture / Climate preferred

- Communication prospects

- Training and learning

\section{Analysis and Discussion of the Results}

\subsection{The Process of Data Collection}

To guide our study and provide the answers to our problematic we chose to adopt the qualitative approach. The choice is justified by the nature of our work, which consists of a numerical evaluation of the last 4 ITEF master classes at the Ben M'sik faculty. 


\subsection{The choice of the University as a field of Investigation}

The use of e-assessment in Morocco is limited at company level. The choice of the university as a field of investigation is justified by a perspective to introduce innovative methods and practices that allow to be part of a quality approach, by the evaluation of the laureates and consider them as a final product close to integrate the professional world.

We chose the students of the Master ITEF to define the field of our study. The initial number of candidates to be evaluated was 200 students, but we received only 120 candidates.

DISC Index - Englishn $=120$ Females $=51.5$ Males $=48.5 \% \quad 1-$ Nov- 18

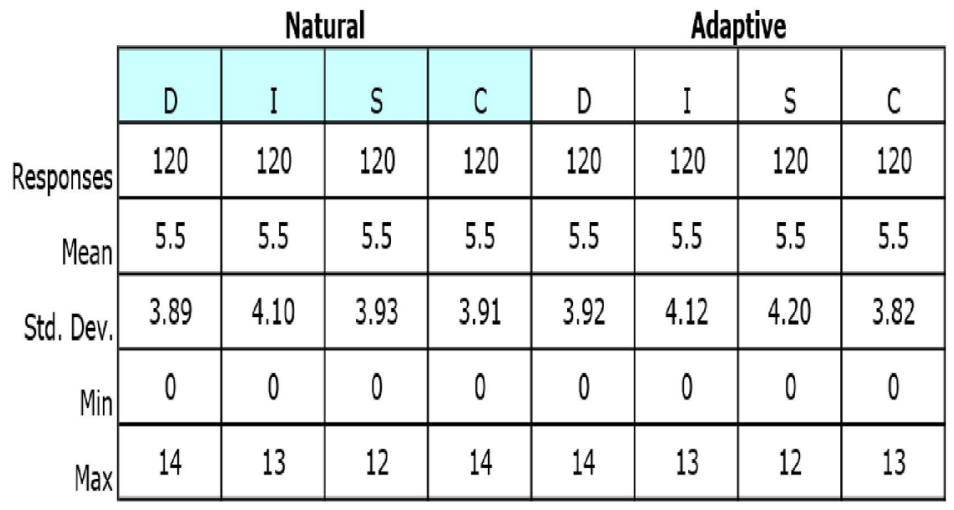

Figure 4. Comparison of Natural and Adapted Styles

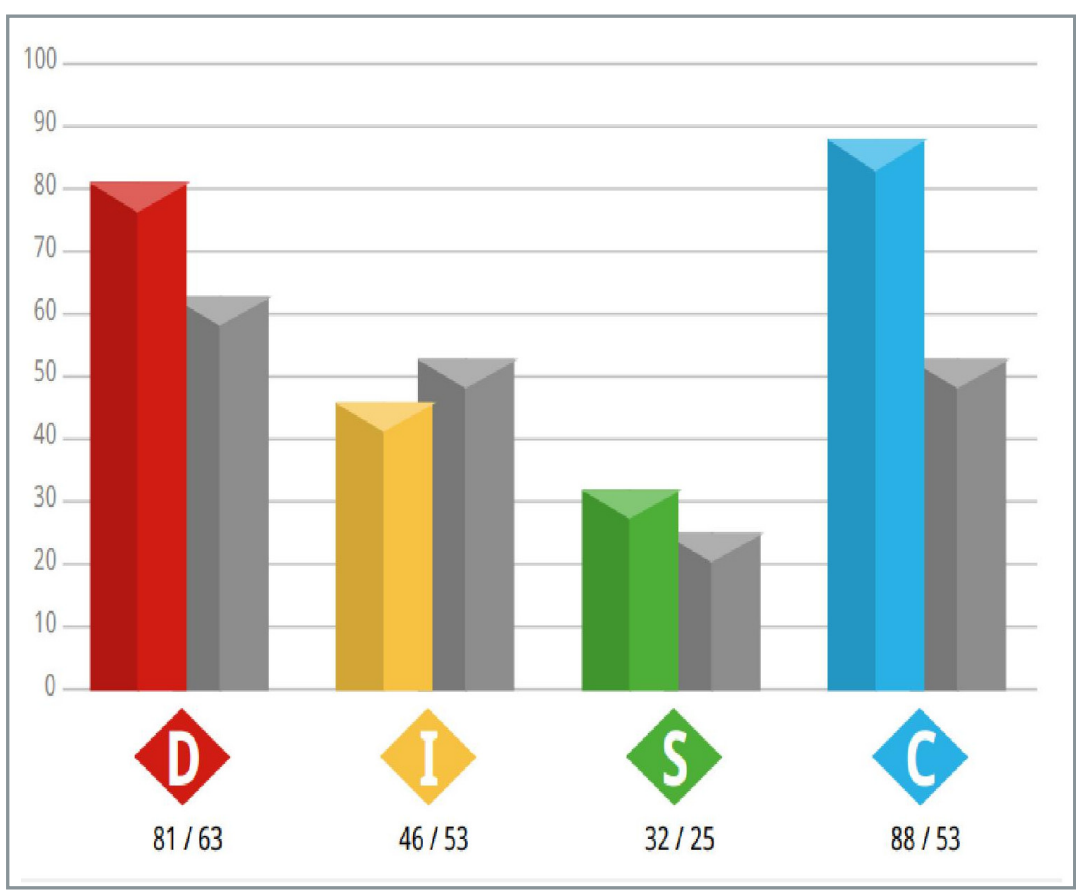

The numerical evaluation of the students allowed us to have a typology of skills and to have a comparison between the natural and adapted style. The graphs below show the characteristics and the dominant personality trait of the candidates.

Dominance: According to the histogram obtained we see a high rate of D which amounts to $\mathbf{8 1 \%}$. This means that the assessed candidates tend to solve new problems very quickly and with confidence. They take an active and direct approach to achieving results. The main thing here is the new problems, those that are unprecedented or have never appeared before. There may also 


\section{Cronbach's Alpha (a) reliabilities}

\begin{tabular}{|l|l|l|}
\hline Scale & Natural & Adaptive \\
\hline Decisive & 0.81 & 0.63 \\
\hline Interactive & 0.46 & 0.53 \\
\hline Stability & 0.32 & 0.25 \\
\hline Cautious & 0.88 & 0.53 \\
\hline
\end{tabular}

Table 2. Cronbach's Alpha (a) reliabilities

be an element of risk in adopting a wrong approach or developing an incorrect solution, but those with a high $\mathrm{D}$ score are eager to take those risks, even if they may be incorrect.

The Influence: The rate perceived on the graph is $\mathbf{4 6 \%}$ which has a low rate compared to the norm, it means that the laureates tend to meet new people in a more controlled, calm and reserved way. This is where the keyword "new people" enters the equation. Those with Low I scores are loquacious with their friends and close associates, but tend to be more reserved with the people they have just met. They tend to focus on controlling emotions, and to approach new relationships with a more thoughtful than affective approach.

Stability: the histogram represents a low rate of stability that rises to $\mathbf{3 2} \%$, this shows that students have a preference to work in a more flexible, dynamic, and unstructured. They value freedom of expression and the ability to move quickly from one activity to another. They tend to get bored with the same routine that provides security to those with a High S.

The $\mathrm{C}$ in the DISC stands for Compliance. The score on the scale shown below shows a high score of $\mathbf{8 8 \%}$. This rate means that assessed candidates tend to respect the rules, standards, procedures, and protocols set by those who hold the authority and

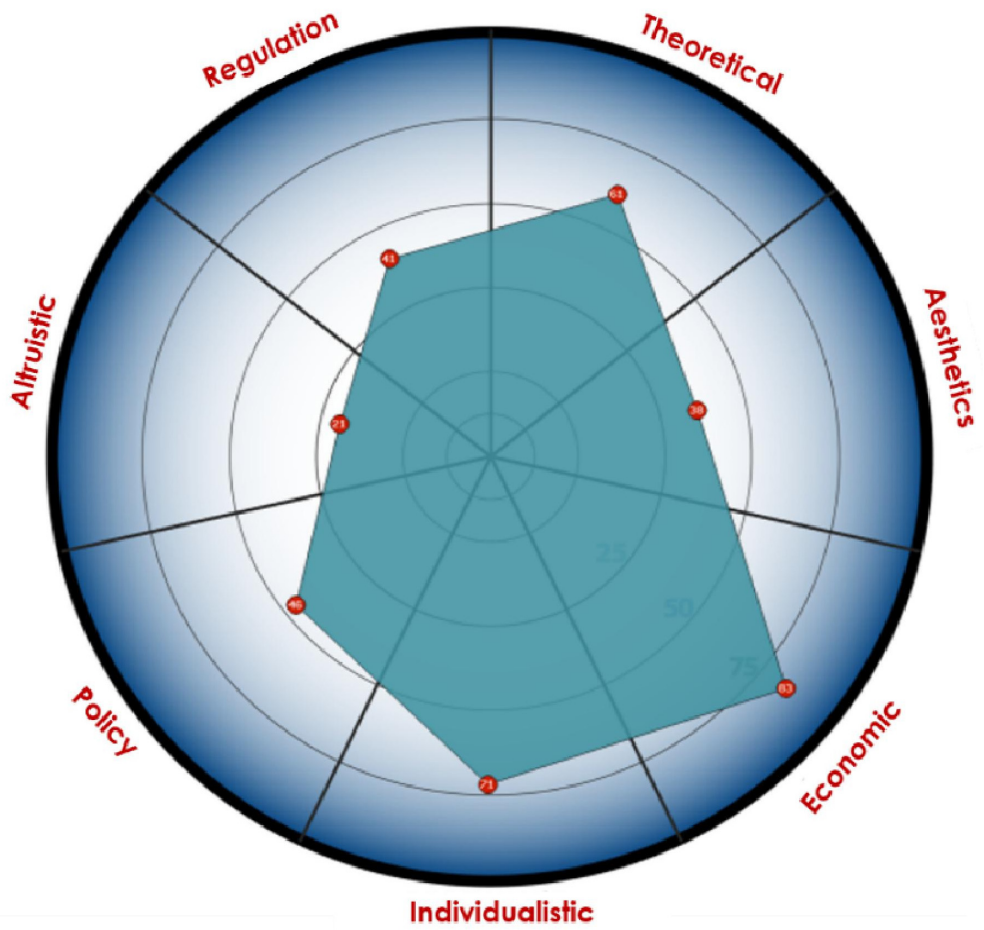

Figure 5. Mapping dimensions of Value and Motivation 
who respect them. They like things to be done correctly according to the user manual. "Rules are made to be followed" is an appropriate currency for those with high $\mathrm{C}$ scores. They have some of the highest quality control interests of any style and often want others to do the same.

The graphical representation is based on the frequency equivalence of the respondent's dimensional values with similar values in a standardized population.

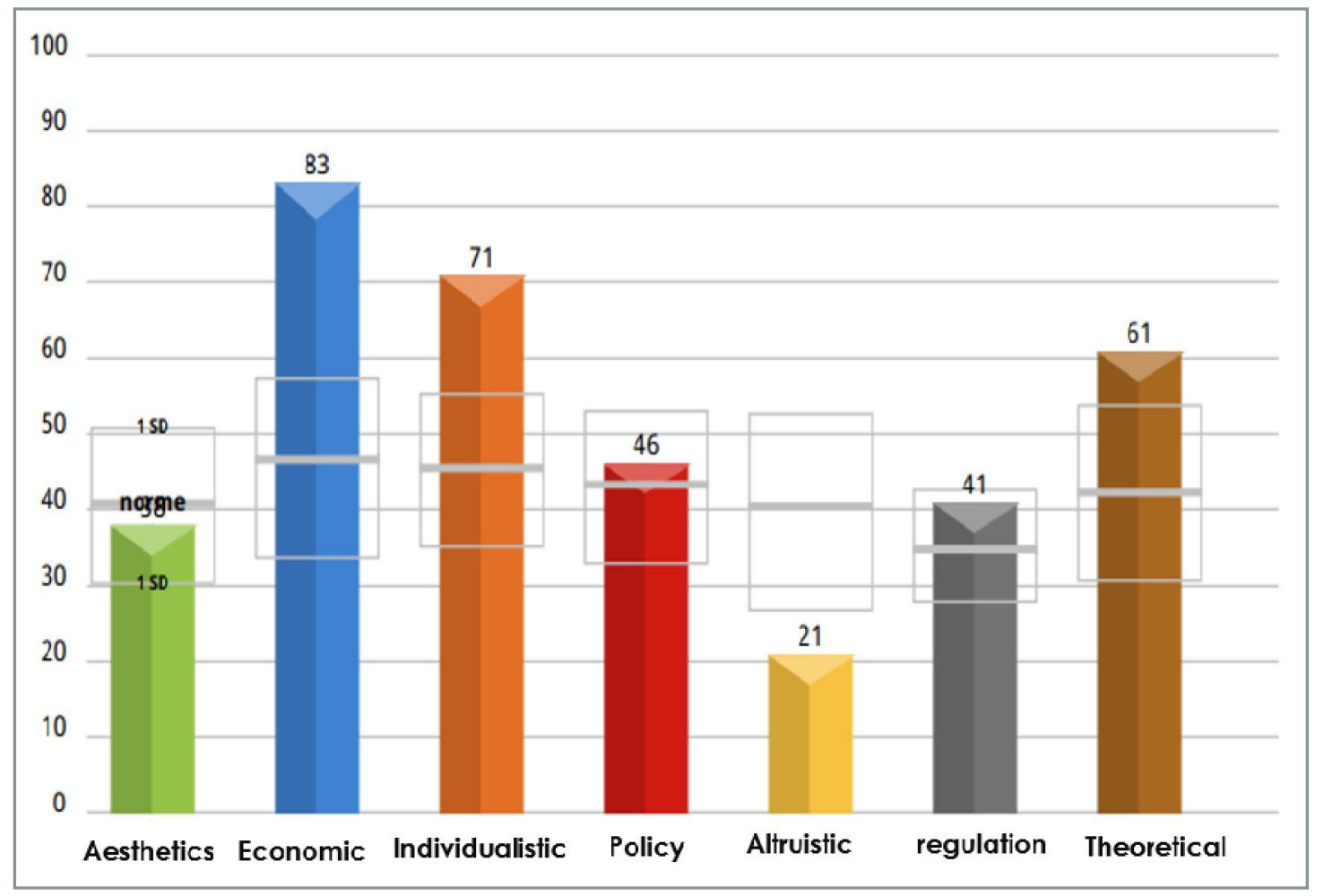

Figure 5. General Summary of Student Values

-Average Aesthetics: Harmony average motivation for balance and form.

-Very high Economic: A motivation for economic and concrete returns.

-Very high Individualistic: A desire to stand out as an independent and unique person.

-Average Policy: Average desire to control or exert influence.

-Low Altruistic: A weak tendency to make humanitarian efforts or to help others concretely and selflessly.

-Average regulation: Propensity to establish order, routine and structure.

-High Theoretical: A willingness to learn, understand and acquire knowledge.

\section{Conclusion}

Universities train students with an entrepreneurial spirit, stimulating their development so that they know, with a global vision, see the opportunities and know how to face the uncertainties, risks and failures they may be exposed to in the world today.

In this perspective the university needs powerful tools to assess the level of development of the student's practical thought, 
creativity and initiative that allow him to identify problems and solutions in a realistic and innovative way. A tool that also measures the level of self-confidence and personal commitment, fundamental to achieving success and goals, always being quality-oriented and tackling with safety and determination possible obstacles to laureates.

In order to achieve this goal, the Moroccan university must be part of a perspective of use of e-assessment platforms taking advantage of a very powerful multi-agent system, which allows to have a cartography and a precise measurement of the competences of the winners. The relevance of its platforms is not limited to evaluation, but they play a role of a training center and digital orientation for the benefit of educational institutions and for the benefit of students. This role ensures continuous development over time, building on gaps and results.

\section{References}

[1] Di Bitonto, P., Laterza, M., Roselli, T., Rossano, V. (2010). An Evaluation Method for Multi-Agent Systems. In: Jêdrzejowicz, P., Nguyen, N. T., Howlet, R. J., Jain, L. C. (eds.) KES-AMSTA 2010. LNCS, 6070, 32-41.

[2] Hendrickx, J., Martin, S. (2016). Open multi-agent systems: Gossiping with deterministic arrivals and departures. In: 54th Annual Allerton Conference on Communication, Control, and Computing.

[3] Bansal, P., Kockelman, K. M., Singh, A. (2016). Assessing public opinions of and interest in new vehicle technologies: An Austin perspective. Transportation Research Part C: Emerging Technologies, 67, 1-14.

[4] Varma, V., Morarescu, I. (2017). Modeling stochastic dynamics of agents with multi-leveled opinions and binary actions, In: Proc. of the $56^{\text {th }}$ IEEE Conference on Decision and Control.

[5] Gutiérez, C., García-Magariño, I., Gómez-Sanz, J. J. (2009). Evaluation of Multi-Agent System Communication in INGENIAS. In: Cabestany, J., Sandoval, F., Prieto, A., Corchado, J. M. (eds.) IWANN 2009. LNCS, 5517, 619-626.

[6] Stocker, A., Shaheen, S. (2018). Shared Automated Mobility: Early Exploration and Potential Impacts, In: Road Vehicle Automation, 4, 125-139.

[7] Xu, J., Tekin, C., Zhang, S., Van der Schaar, M. (2015). Distributed multi-agent online learning based on global feedback, IEEE Transactions on Signal Processing, 63 (9) 2225-2238.

[8] Wooldridge, M., Jennings, N. R. (1995). Intelligent agents: Theory and practice. The Knowledge Engineering Review, 115152.

[9] Wandel, A. P., Robinson, C., Abdulla, S., Dalby, T., Frederiks, A., Galligan, L. (2015). Students' mathematical preparation: Differences in staff and student perceptions. I. J. of Innovation in Science and Math. Education, 23(1).

[10] Vojak, C., Kline, S., Cope, B., McCarthey, S., Kalantzis, M. (2011) New spaces and old places: An analysis of writing assessment software. Computers \& Composition, 28, 97-111.

[11] Foltz, P. W., Laham, D., Landauer, T. K. (1999). Automated Essay Scoring: Applications to Educational Technology. In: Proceedings of EdMedia '99.

[12] Aven, T. (2016). Ignoring scenarios in risk assessments: understanding the issue and improving current practice. Reliab Eng Syst Safvol 20, 145 - 215. 